\title{
El autoconcepto en emprendedores
}

José Miguel Giménez Lozano, Diego Candela Salomón, Macarena Erviti Lara, Juan Carlos Castillo Bautista y Javier Delgado Asenjo. Universidad de Granada

Recepción: 31 de mayo de 2016 | Revisión: 15 de junio de 2016 | Aceptado: 12 de julio de 2016

Correspondencia: josemi1992@correo.ugr.es

Citar: Giménez, J.M., Candela, D., Erviti, M., Castillo, J.C. y Delgado, J. (2016). El autoconcepto en emprendedores. ReiDoCrea, 5(2), 71-77.

Resumen: Las características de la población emprendedora están siendo muy estudiadas en la actualidad. Objetivo: El objetivo de esta investigación ha sido estudiar el autoconcepto entre una población emprendedora comparándola con un grupo de no emprendedores activos laboralmente del área de Granada, e intentar descubrir si esa motivación emprendedora fue impulsada por experiencias negativas en el ámbito laboral. Método: Contamos con un número total de 67 participantes, divididos en emprendedores $(N=34)$ y no emprendedores $(N=33)$. A ambos grupos se les aplicó el cuestionario de evaluación del autoconceptoAF-5, un cuestionario sociodemográfico y una pregunta sobre experiencias negativas laborales. Resultados: Se hallaron resultados significativos en emprendedores en comparación con los no emprendedores, en una de las cinco dimensiones que se miden: el autoconcepto emocional. Conclusiones: El autoconcepto emocional es vital para un emprendedor ya que aporta mayor seguridad a la persona que se debate entre comenzar su propio proyecto laboral o no.

Palabras clave: Trabajador Independiente | Psicología Ocupacional

\section{Self-Concept in Entrepreneurs}

\begin{abstract}
The characteristics of the entrepreneur population are being investigated currently. Objective: The aim of this investigation was to study the self-concept a entrepreneur's population comparing with an ocupationally active nonentrepreneurs group, in the area of Granada, and try to discover if that entrepreneur motivation was inspired by negative experiences in the working sphere. Method: We had a total number of 67 participants, divided in entrepreneurs $(\mathrm{N}=34)$ and non-entrepreneurs $(\mathrm{N}=33)$. Both groups were applied the AF-5 self-concept questionnaire, a sociodemographic questionnaire and a question about negative working experiences. Results: Significant results were found in entrepreneurs in comparison with non-entrepreneurs in one of the five dimensions measured: the emotional selfconcept. Conclusions: The emotional self-concept is vital for an entrepreneur as provides more security to the person who wavers between start his own project or not.
\end{abstract}

Keywords: Self-employed | Occupational Psychology

\section{Introducción}

Actualmente, la actitud y las habilidades emprendedoras están siendo muy estudiadas (Esnaola, Goñi y Madariaga, 2008). No es difícil encontrar estudios que hagan referencia a algún tipo de característica de la personalidad o social relacionada con los individuos emprendedores; se pueden encontrar investigaciones tanto desde un punto de vista de la teoría de rasgos, como desde un punto de vista más psicosocial.

Concretamente, en España, según datos del informe GEM-España (Global Entrepreneurship Monitor España), en 2014 la tasa de actividad emprendedora (TEA) supuso un $5,47 \%$ de la población general con una edad media de 40 años, situando así, el $31,2 \%$ entre 35 y 44 años, de los cuales el $47,6 \%$ de los emprendedores tenían Formación Superior o de Postgrado. Además, 6 de cada 10 eran hombres. En comparación con otros países de la UE, España, tienen una TEA muy parecida, un porcentaje similar al de Francia y Alemania, ambos con un $5,3 \%$ de TEA. Pero, a su vez, queda muy por debajo del Reino Unido con un $8,6 \%$ y de EEUU con un $13,8 \%$ (Legazkue et al., 2015). El objetivo de nuestro estudio es comparar el autoconcepto de la población activa que no dirige una empresa con la población emprendedora.

Los conceptos básicos de esta investigación son el autoconcepto y la actitud emprendedora. Se entiende actitud emprendedora como la acción generada por las personas que tienen la habilidad de percibir una oportunidad y a partir de ella crear un 
negocio, que le permita el sustento económico. Fenómeno que puede posibilitar la superación de las dificultades económicas de las personas y a la vez puede conllevar el crecimiento económico de la sociedad (Latorre y Vanessa, 2011).

Por otra parte, Shavelson, Hubner y Stanton (1976), definen el autoconcepto como la percepción que el individuo tiene de sí mismo generada en torno a las experiencias con su entorno y a las atribuciones que hace de su propia conducta. Plantean así un modelo jerárquico y multifacético que comprende componentes emocionales, laborales (o académicos), físicos, sociales y familiares. Schimitt-Rodermundo y Vondracek (2002) en una investigación que realizaron, encontraron que la conducta emprendedora correlaciona positivamente con la autoeficacia. Además, encontraron que los emprendedores poseen habilidades de autorregulación intencionales. Poon, Ainuddin y Junit (2006) demostraron que las personas emprendedoras tienen altos niveles de necesidad de logro que predispone a la gente a participar en las actividades empresariales y altos niveles de locus de control. Por tanto, aquellos emprendedores que posean un locus de control interno tendrán más persistencia en obtener resultados favorables porque creen que ellos poseen el control sobre dichos resultados, todo lo contrario, a aquellos que tengan un locus de control externo.

Tener un autoconcepto positivo es muy importante en el funcionamiento tanto social como profesional del individuo, esto hace que sea uno de los principales puntos a tratar en una intervención psicológica (Esnaola et al., 2008). Un déficit en el autoconcepto podría generar problemas emocionales y de conducta a corto y a largo plazo. Bajos niveles de autoconcepto, además, se han relacionado con un aumento de la agresividad y del comportamiento antisocial (Baumeister et al., 2003) que pueden perdurar a lo largo de toda la vida.

Se ha comprobado que los motivos por los que la mayoría de personas toman la decisión de emprender son: motivos de oportunidad y motivos de necesidad (Reina Rubiano, E., 2014). Las motivaciones de necesidad se caracterizan por lo deseos de obtener independencia económica, obtener un lugar de trabajo y superar una experiencia laboral negativa. Así las experiencias laborales negativas pueden fomentar que una persona tome la decisión de emprender. Fernández et al. (2012) afirma que un individuo tiene más propensión a crear su propia empresa cuando tiene experiencias laborales negativas en el trabajo.

Así, este trabajo se centra en el autoconcepto en personas emprendedoras. Al hablar de autoconcepto son muchos los puntos de vista que defienden cómo se llega a la actitud emprendedora. Está la Teoría de los Rasgos que defiende que los individuos nacemos con unos rasgos de personalidad innatos e invariables y que la personalidad emprendedora, por tanto, se compone de unos rasgos específicos. Por otro lado, la Teoría del Comportamiento Organizacional, afirma que la personalidad se crea dependiendo de cómo influya el ambiente en la persona y de cómo esta se comunique con la organización o el mismo ambiente (House et al., 1996).

Geldhof (2014) en contra de la creencia del emprendedor como una característica estable de la personalidad, argumenta que el emprendedor emerge de una relación bidireccional entre el individuo activo y las relaciones con otros individuos teniendo en cuenta en el contexto en las que se dan esas relaciones, dando lugar a las Teorías del Desarrollo de Sistemas Relacionales que defienden que los atributos que posee la personalidad de un individuo son importantes para el desarrollo de su carrera como empresario y, por tanto, como emprendedor, pero que dichos atributos funcionan en un sistema de individuo-contexto muy amplio. Los factores ambientales del individuo 
son tan importantes para el desarrollo emprendedor como lo podrían llegar a ser otros más intrínsecos.

Así, el objetivo de esta investigación, es analizar si existen diferencias de autoconcepto entre personas que han creadosu propio negocio y personas no emprendedoras pero activas laboralmente. Para esto, nos hemos acogido al Modelo Multidimensional del Autoconcepto de Shavelson, proponiendo que un emprendedor poseerá mayores puntuaciones en alguna de las cinco dimensiones (emocionales, laborales o académicos, físicos, sociales y familiares) en comparación con un grupo activo laboralmente y no emprendedor (hipótesis 1). Por otro lado, planteamos que la población emprendedora haya sufrido un mayor número de experiencias negativas en comparación con el grupo activo laboralmente y no emprendedor, y que esto les haya motivado para formar su propia empresa (hipótesis 2).

\section{Método}

\section{Participantes}

En este estudio participaron un total de 67 personas de edades comprendidas entre los 19 y 58 años $(M=35,76$; $S D=10,66)$; todos ellos españoles. De esta muestra, 34 eran emprendedores (Edad: $M=37,8 ; S D=8,61$ ) y 33 no emprendedores (Edad $M=33,6 ; S D=12,2$ ). De la muestra total 40 eran varones y 27 mujeres. Todos ellos fueron informados y dieron su consentimiento previamente.

\section{Procedimiento}

La recogida de información se realizó en los meses de octubre, noviembre y diciembre, en la provincia de Granada. La selección de la muestra siguió los siguientes criterios: Para la selección de individuos emprendedores, se eligieron personas a la cabeza de empresas con menos de dos años de antigüedad. En cuanto al grupo de no emprendedores se optó por personas con experiencia laboral que nunca habían creado y dirigido una empresa. La recogida de datos se realizó mediante la entrega directa de los cuestionarios a los individuos seleccionados.

\section{Instrumentos}

La recogida de datos se ha llevado a cabo a través del Autoconcepto Forma 5 (AF-5). El AF-5 es uno de los cuestionarios más utilizados para evaluar el autoconcepto, de fácil aplicación, corrección e interpretación compuesto por 30 elementos que tienen como finalidad evaluar los diferentes aspectos que componen el autoconcepto (Emocional, Físico, Familiar, Académico/profesional y Social) en niños, adolescentes y adultos a partir de los 10 años. Posee una consistencia interna que oscila entre $.71 \mathrm{y}$ .88, con una fiabilidad de .84 (García y Musitu, 2014).Además, se ha adjuntado un breve cuestionario sociodemográfico que incluye preguntas frecuentes que hacen referencia a la edad, al sexo, al nivel de estudios del sujeto, al de sus padres y si han tenido alguna experiencia laboral negativa.

\section{Resultados}

Los datos fueron recogidos y introducidos en el programa estadístico SPSS, para su análisis. La aplicación y corrección se llevó a cabo bajo las instrucciones generales y normas del AF-5. 
En un análisis de medias y desviaciones típicas nos muestran que un 34 de los sujetos encuestados eran emprendedores y un 33 no emprendedores, con una edad media total de 35, $76(D T=10,63)$. Siendo la edad media de los emprendedores 37,79 $(D T=8,61)$ y la edad media de los no emprendedores $33,67(D T=12,20)$. En la división por sexos se encuestaron a 40 hombres, de los cuales 21 eran emprendedores $(52,5 \%$ de sujetos masculinos), y 27 mujeres, de las cuales 13 eran emprendedoras $(48,1 \%$ de los sujetos femeninos). Véase Tabla 1.

\begin{tabular}{|c|c|c|c|c|c|}
\hline \multicolumn{6}{|c|}{ Tabla 1. Estadísticos descriptivos de la muestra } \\
\hline & & Frecuencia & Porcentaje & Edad Media & D.T. edad \\
\hline \multirow[t]{2}{*}{ Emprendedores } & Mujeres & 13 & $52.5 \%$ & 37.79 & 8.61 \\
\hline & Hombre & 21 & $48.1 \%$ & & \\
\hline \multirow[t]{2}{*}{ No emprendedores } & Mujeres & 14 & $51.9 \%$ & 33.67 & 12.20 \\
\hline & Hombres & 19 & 47.5 & & \\
\hline
\end{tabular}

En segundo lugar, se obtuvo de cada participante las puntuaciones en las respectivas dimensiones medidas por el cuestionario (autoconcepto académico, emocional, social, familiar y físico) y se compararon mediante la prueba T con un intervalo de confianza del 95\%. Véase Tabla 2.

\begin{tabular}{|c|c|c|c|c|c|c|}
\hline \multicolumn{7}{|c|}{ Tabla 2.Puntuaciones autoconcepto por grupos. } \\
\hline & Em & & No emprendedor & & & \\
\hline Autoconcepto & Media & D.T & Media & D.T & t & $P$ \\
\hline Académico & 8,35 & 1,21 & 7,89 & 1,27 & 1,54 & ,13 \\
\hline Social & 7,52 & 1,65 & 7,15 & 1,57 & ,95 & ,34 \\
\hline Emocional & 6.66 & 1.70 & 5.28 & 1,80 & 3,2 & ,002 \\
\hline Familiar & 8,54 & 1,28 & 7,72 & 1,63 & 2,3 & ,025 \\
\hline Físico & 6,166 & 2,20 & 5,80 & 1,72 &, 76 & ,45 \\
\hline
\end{tabular}

A partir de la tabla, vemos una serie de diferencias no significativas en la mayoría de dimensiones. No obstante, los emprendedores sí muestran un mayor autoconcepto emocional que los no emprendedores $\left(t_{(64,56)}=3,229, p<0,05\right.$.

Entre otros resultados, como prueba exploratoria hicimos pruebas $\mathrm{T}$ con intervalo de confianza a los autoconceptos, según la variable sexo. Cabe destacar el hallazgo de diferencias significativas entre hombres y mujeres tanto en autoconcepto físico $\left(t_{(65)}=2,104, p<0,05\right)$ como emocional $\left(t_{(65)}=2,761, p<0,05\right)$; Véase Tabla 3. Sin embargo, en el resto de dimensiones evaluadas no se informa de ninguna diferencia significativa.

\begin{tabular}{|lcccccc|}
\hline \multicolumn{7}{c|}{ Tabla 3. Puntuación autoconcepto por sexo } \\
\hline \multicolumn{7}{c}{ Hombre } \\
Media & D.T & Media & D.T & t & P \\
\hline Autoconcepto & 8,11 & 1,18 & 8,14 & 1,37 &,- 066 &, 95 \\
\hline Académico & 7,49 & 1,61 & 7,11 & 1,61 &, 96 &, 34 \\
Social & 6,48 & 1,63 & 5,25 & 1,99 & 2,76 &, 007 \\
Emocional & 8,27 & 1,38 & 7,93 & 1,69 &, 92 &, 36 \\
Familiar & 6,39 & 1,74 & 5,39 & 2,17 & 2,1 &, 039 \\
Físico & & & & & & \\
\hline
\end{tabular}


Por otra parte, se ha llevado a cabo una prueba Chi-cuadrado para comparar las experiencias laborales negativas de la muestra emprendedora con las de la muestra no emprendedora. No informamos de diferencias significativas.

\section{Discusión}

En cuanto a los resultados obtenidos desde una muestra de emprendedores similar a la media extraída del GEM tanto en edad media como en formación, se encuentra un mayor autoconcepto emocional en la muestra emprendedora frente a la no emprendedora, datos que apoyan la hipótesis principal. Si bien se esperaba hallar una diferencia más acentuada en prácticamente la totalidad de las dimensiones, es la dimensión emocional la que se considera que aporta mayor seguridad a la persona que se debate entre comenzar su propio proyecto laboral o no.

Un autoconcepto emocional alto se traduce en un mayor control de las emociones y situaciones. Correlaciona con una aproximación adecuada y con menor nerviosismo a los diferentes momentos de nuestra vida. Se atribuye lo contrario a un autoconcepto emocional bajo. Así, el autoconcepto emocional correlaciona positivamente con el autocontrol, las habilidades socia les, la sensación de bienestar y la aceptación de los iguales. (Cavaet al. 2000). Este auto concepto emocional alto también correlaciona con un locus de control interno tal y como afirman Poon et al. (2006). Es decir, estos emprendedores, percibirían que la responsabilidad en las consecuencias de sus acciones recae en ellos y por ello se situarían en posiciones de tomas de decisiones en comparación con los no emprendedores. Esto supondría que un fracaso en la toma de decisiones de su proyecto laboral podría ponerlos en riesgo y afectar a su autoconcepto emocional positivo con una alta probabilidad (Baron et al. 2012).

De esta forma, una persona con mayor seguridad propia tendrá mayor probabilidad de decantarse por un proyecto liderado por sí misma, tomando una decisión considerada comúnmente arriesgada por las diversas implicaciones económicas que conlleva (Baron, A. et al. 2012).Y así, las personas con un autoconcepto emocional negativo, que correlacionan con sintomatología depresiva, ansiedad y pobre integración en el ámbito laboral, abogarán por otro tipo de salidas laborales percibidas como menos arriesgadas.

En cuanto a las diferencias significativas encontradas en autoconcepto emocional y físico entre hombres y mujeres, sorprende que, estando proporcionada la cantidad de emprendedores en la muestra en ambos sexos, la muestra de mujeres presente un menor autoconcepto tanto como físico como emocional. Así pues, estas diferencias se podrían explicar realizando futuras investigaciones comparando tanto el autoconcepto emocional como el autoconcepto físico en las cuatro categorías de la muestra (sexo: hombre y mujer y actitud emprendedora y no emprendedora), estos estudios revelarían que las dos dimensiones del autoconcepto serían similares tanto para la mujer como para el hombre emprendedor, pero significativamente distintos para el hombre y la mujer no emprendedora. Lo cual implicaría que no hay diferencias tan grandes entre el hombre emprendedor y no emprendedor en lo que a los autoconceptos mencionados se refiere e incluso que estas no fueran significativas, de ahí que surgirían una serie de cuestiones en torno al porqué un hombre se decide a emprender más allá del autoconcepto.

Por otra parte, la ausencia de diferencias significativas entre emprendedores y no emprendedores en experiencias laborales negativas no apoya la segunda hipótesis. Esto puede deberse a que la muestra no es lo suficientemente grande porque entra en conflicto con lo propuesto por Fernández et al. (2012). 
Para concluir, cabe resaltar las diferencias significativas en el autoconcepto emocional en comparación con el grupo emprendedor y no emprendedor, así como destacar también que no se han hallado diferencias significativas en relación con las experiencias laborales negativas en emprendedores con respecto a no emprendedores. Ya se ha hablado de las implicaciones que conlleva el pequeño tamaño de la muestra utilizada en esta investigación. Por tanto, en posteriores investigaciones, se tratará de resolver esta cuestión.

Este problema no solo afecta a las hipótesis propuestas, sino que afecta prácticamente a la totalidad del estudio. Una mayor muestra hubiera aportado mayor homogeneidad entre los dos grupos sujeto de estudio y una mayor probabilidad de concluir en resultados cercanos a la literatura del campo. También tener en cuenta la escasez en profundidad a la hora de ahondar en las experiencias negativas, es decir, profundizar en qué tipo de experiencias negativas han sufrido, cómo de intensas las han percibido, y si eso les ha motivado para abandonar su trabajo. Investigaciones futuras deberían estudiar una muestra homogénea en referencia al sector al que se dedican, pues en esta investigación la naturaleza de los sectores es muy diversa. Por último, es también conveniente destacar aspectos metodológicos de los que no hayamos sido conscientes durante la realización de este trabajo.

Finalmente, este estudio ha intentado aproximarse a las diferencias entre emprendedores y no emprendedores, descubriendo que una parte muy importante para crear un negocio es que la persona tenga un buen concepto emocional, una autoestima alta.

\section{Referencias}

Baron, R.A., Hmieleski, K.M., \& Henry, R.A. (2012). Entrepreneurs' dispositional positive affect: The potential benefits - and potential costs - of being "up". Journal of Business Venturing, 27, 310-324

Baumeister, R.F., Campbell, J.D., Krueger, J.I., \& Vohs, K.D. (2003). Does high self-esteem cause better performance, interpersonal success, happiness, or healthier lifestyles? Psychological Scienceinthe Publiclnterest, 4, 1-44.

Byrne, B.M., \& Shavelson, R.J. (1996). On the structure of social self-concept for pre-, early, and late adolescents: A test of the Shavelson, Hubner, and Stanton (1976) model. Journal of Personality and Social Psychology, 70(3), 599-613.

Byrne, B.M., \& Shavelson, R.J. (1986). On the structure of adolescent self-concept. Journal of EducationalPsychology, 78(6), 474.

Cava, M.J., Musitu, G. y Vera, A. (2000). Efectos directos e indirectos de la autoestima en el ánimo depresivo. Revista Mexicana de Psicología, 17(2), 151-161.

Esnaola, I., Goñi., A. y Madariaga J. M. (2008). El autoconcepto: perspectivas de investigación. Revista de Psicodidáctica, 13(1),179-197.

Fernández, M.L., Fernández, P.M.R. y Carrión, R.D. (2012). Motivaciones para emprender: un análisis de diferencias entre hombres y mujeres. Economía industrial, (383), 75-84.

Geldhof, J., Malin, H., \& Johnson, S.K. (2014). Entrepreneurship in young adults: Initial findings from the young entrepreneurs study. Journal of Applied Developmental Psychology, 35, 410-421

House, R. J., Shane, S. A. yHerold,D. M. (1996) 'Rumors of the Death of Dispositional Research are Vastly Exaggerated', Academy of Management Review 21(1), 203-24.

Latorre, M. y Vanessa, Z. (2011). La motivación, el autoconcepto y la creatividad, como factores esenciales para el emprendimiento. Universidad católica de Pereira, Risaralda, Colombia.

Legazkue, I.P., Guerrero, M. y González-Pernía, J.L. (2015). Global Entrepreneurship Monitor: Informe GEM España 2014 (Vol. 18). Ed. Universidad de Cantabria. 
Reina Rubiano, E. (2014). Emprendimiento y liderazgo. Bogotá: Escuela de Administración Bogotá D. C.

Shavelson, R.J., Hubner, J.J., \& Stanton, J.C. (1976). Self concept: Validation of construct interpretations. Review of Educational Research, 46(3), 407-441.

Shavelson, R.J., Burstein, L., \& Keesling, J.W. (1977). Methodological considerations in interpreting research on self-concept. Journal of youth and adolescence, 6(3), 295-307. 